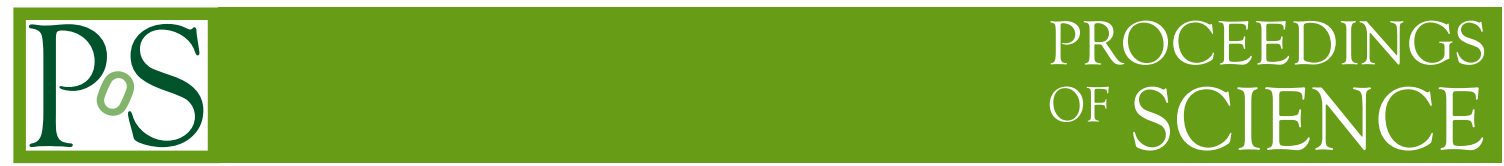

\title{
CMS RPC Activities During LS2 and Commissioning
}

\section{Barroso on behalf of the CMS Collaboration ${ }^{a, *}$}

${ }^{a}$ Universidade do Estado do Rio de Janeiro,

São Francisco Xavier Street 524, Rio de Janeiro, Brazil

E-mail: mapse@cern.ch

The second LHC long shutdown period (LS2) was a crucial opportunity to complete the consolidation and upgrade projects for the CMS Resistive Plate Chambers (RPCs). The consolidation included detector maintenance for gas tightness, HV (high voltage), LV (low voltage) and slow control operation. Dedicated studies were performed to understand the behaviour of RPC currents with comparison to Run 2. This paper summarises the activities performed and commissioning of CMS RPC on the surface (For RE4) and for full detector in CMS cavern in different operating conditions.

The Ninth Annual Conference on Large Hadron Collider Physics - LHCP2021

7-12 June 2021

Online

${ }^{*}$ Speaker 


\section{Introduction}

The CMS [1] muon system uses three different types of detector technologies [2]: cathode strip chambers (CSC) in the endcap region, drift tubes (DT) in the barrel region, and resistive plate chambers (RPC) in both regions. The RPC system contributes to muon identification, reconstruction and triggering. The barrel region is divided into 5 wheels, called $\mathrm{W} \pm 2, \mathrm{~W} \pm 1, \mathrm{~W} 0$, while the endcap region is divided in 8 regions, called $\mathrm{RE} \pm 4, \mathrm{RE} \pm 3, \mathrm{RE} \pm 2, \mathrm{RE} \pm 1$. A wheel consists of 4 muon stations $(R B 1, R B 2, R B 3, R B 4)$ at increasing radius and is divided in 12 sectors in $\phi$, while the endcap is divided in 36 sectors. There are 1056 RPC chambers in total, covering an area of more than $3000 \mathrm{~m}^{2}$. They work in double-gap mode, where each gap is $2 \mathrm{~mm}$ width and each copper strip readout is a plane located between the gas gaps. The gas mixture used is composed of $\mathrm{C}_{2} \mathrm{H}_{2} \mathrm{~F}_{4}$ (95.2\%), $i C_{4} H_{10}(4.5 \%)$ and $S F_{6}(0.3 \%)$ with a relative humidity of 40-50\% [3]. The main purpose of the second LHC Long Shutdown Period (LS2) for the CMS RPC is to allow the completion of the consolidation and upgrade projects.

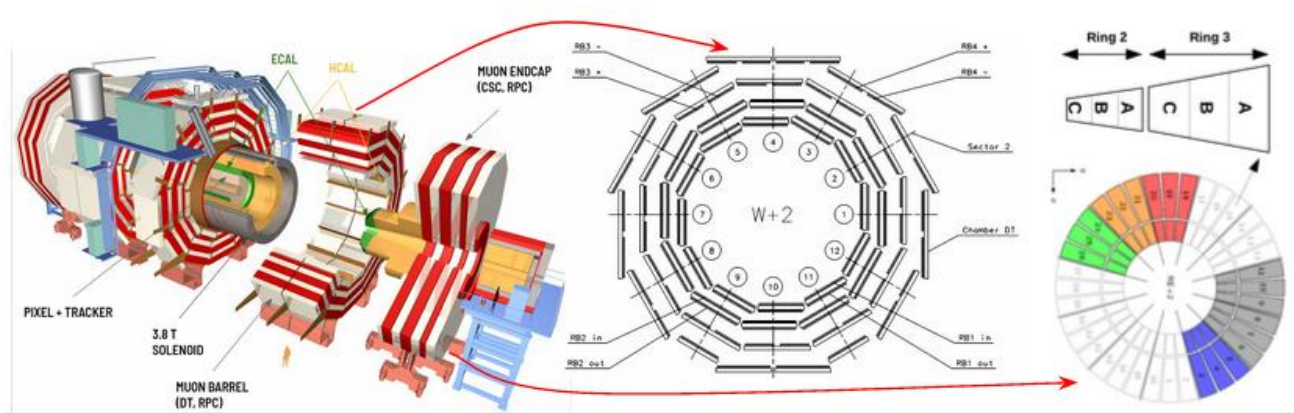

Figure 1: The CMS system: (left) the entire system, showing the tracker, electromagnetic calorimeter (ECAL), hadronic calorimeter (HCAL), muon (CSC, DT, and RPC) subsystems and the $3.8 \mathrm{~T}$ solenoid; (middle) a typical CMS wheel with its respective stations, and (right) a typical endcap station, showing ring 2 and ring 3 of RPC.

\section{RPC gas system repairs}

In the barrel 49 out of 99 gas leaky RPCs were repaired. The Figure (2) shows that the efficiency increased after the repair campaign. After successful repair it was observed a gain in $1.4 \%$ on the average efficiency. Note that each barrel chamber is divided into 2 or 3 eta partitions. In barrel there are 480 chambers installed resulting in 1020 eta partitions called as "rolls".

\section{HV/LV Maintenance}

The applied high voltage is important to keep the RPCs in the working point (WP) to achieve good performance. The goal of the HV maintenance is then to identify which part of the system is showing problems and to fix it with the best possible solution. The aim of the low voltage repairs is to guarantee the powering and required configuration of the front-end board (FEBs). During LS2 a total of $65 \mathrm{HV}$ repairs were done. Besides that, $12 \mathrm{LV}$ repairs were successfully performed. The Figure (3) shows that with the HV maintenance, the average efficiency has increased $6 \%$. 


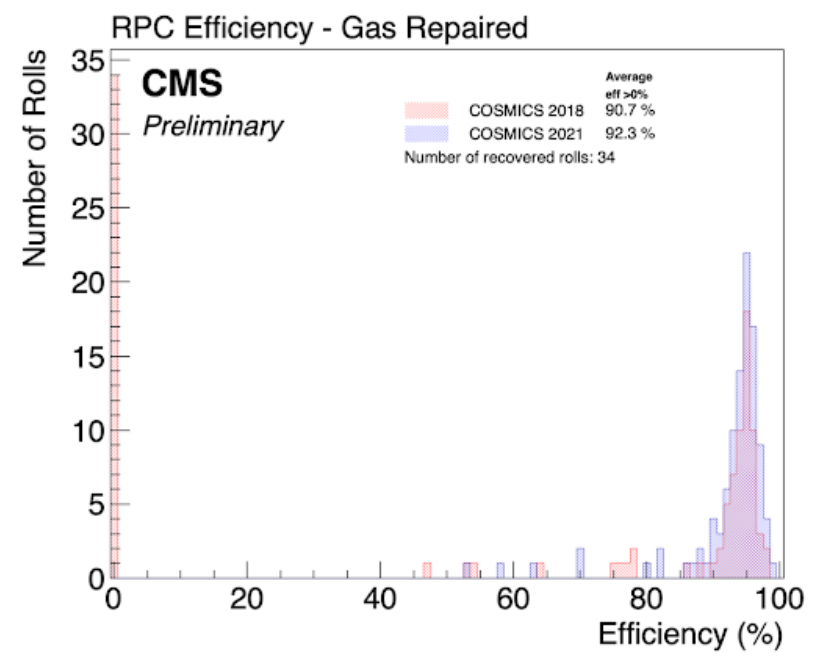

Figure 2: Comparison of the efficiency between 2018 and 2021 for the barrel chambers that were repaired. The total number of rolls recovered is 34 meaning $1.4 \%$ in efficiency gain.

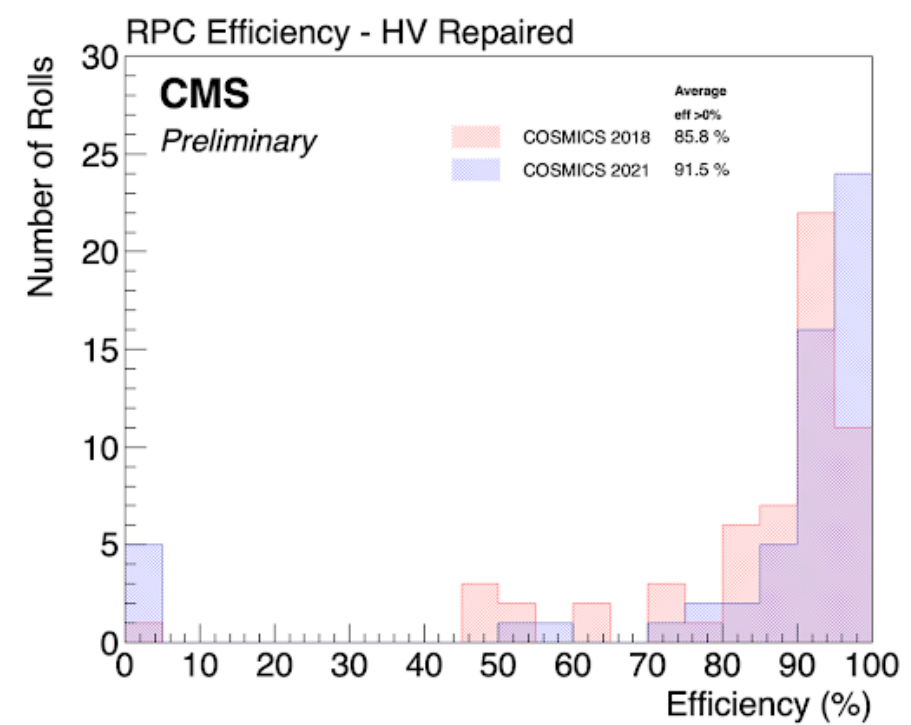

Figure 3: Comparison of efficiency between 2018 and 2021 in the barrel chambers concerning HV repairs. The overall increase in the efficiency is approximately $6 \%$.

\section{RE4 Activities}

The endcap stations $R E \pm 4$ were extracted during LS2 to allow the CSC ME4/1 extraction to replace the electronics. A new laboratory with controlled environmental conditions was built at CMS Point 5 to house the chambers. However, when powered on the surface the currents were found higher than their last values at the end of Run 2. A known source of increase of the current is the $F^{-}$production, in which the higher the concentration of this ion in the gas, greater the current. As the chambers were in air for months, this effect can be discarded, as it takes place in the presence of high backgrounds, which is not the case. So far, there is no explanation for this. However, when chambers are under $\mathrm{HV}$ for long periods, the currents decrease to values comparable to the ones at 
the end of Run 2, as can be seen on Figure (4).

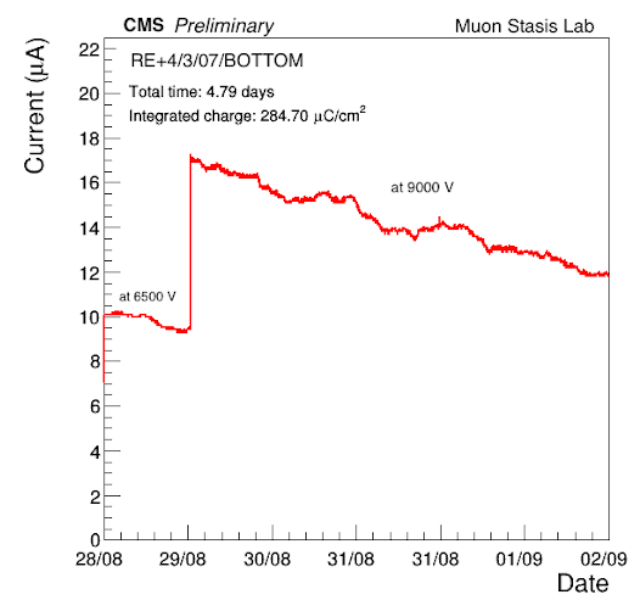

Figure 4: Decrease in the currents after HV conditioning.

\section{RPC Performance}

After all interventions in LS2, the performance of the detector is improved with respect to the end of Run 2. Is worth mentioning that these data were taken with cosmic runs. Also, the cluster size is in accordance with the expected values. Aiming to minimize the environmental impact of the RPC gases, the CERN EPDT gas team have developed the first $\mathrm{C}_{2} \mathrm{H}_{2} F_{4}$ recuperation system with efficiency of $80 \%$ showed on Figure (5). In order to minimize the global warming potential for Run 3, the RPC plan to turn off all the leaky chambers (3.5\% of total system), reducing the gas leaks from 900 to $200 \mathrm{l} / \mathrm{h}$.

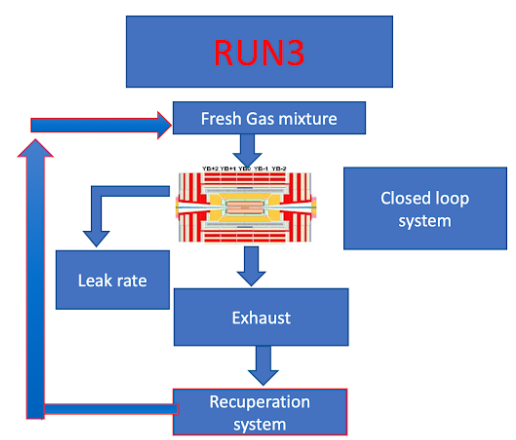

Figure 5: Gas recuperation system. Now instead of throwing all the gas in the environment a percentage of it is recovered.

\section{Conclusion}

After operation in high background conditions during Run 1 and Run 2, the RPCs show a stable performance, with average efficiency around 95\% and average cluster size around 2 strips. The interventions done in LS2 were crucial to improve system's performance, as well as to reduce the RPCs greenhouse gases (GHG) emission in the environment during Run 3 data taking. 


\section{References}

[1] S. Chatrchyan et al. [CMS Collaboration], JINST 3 (2008), S08004 doi:10.1088/17480221/3/08/S08004

[2] CMS COLLABORATION, et al. Technical Design Report. CERN/LHCC, (1997), vol. 97, p. 33.

[3] CMS COLLABORATION, et al. The phase-2 upgrade of the CMS muon detectors. Technical Design Report, 2017. 